\title{
Kaposi's Sarcoma Presenting as Lymphadenopathy in an Immunocompetent Patient
}

\author{
Hana Zoubeidi ${ }^{1}$, Zohra Aydi ${ }^{1}$, Fatma Daoud ${ }^{1}$, Imen Rachdi ${ }^{1}$, Wafa Koubaa ${ }^{2}$, Raja Jouini ${ }^{2}$, Lilia Baili ${ }^{1}$, \\ Achraf Debbiche², Besma Ben Dhaou ${ }^{1}$, Fatma Boussema ${ }^{1}$ \\ ${ }^{1}$ Department of Internal Medicine, Rabta Hospital, Tunis, Tunisia \\ ${ }^{2}$ Anatomopathology Department, Habib Thameur Hospital, Tunis, Tunisia
}

\section{Doi: 10.12890/2016_000493-European Journal of Case Reports in Internal Medicine - @ EFIM 2016}

Received: 30/08/2016

Accepted: 05/09/2016

Published: 16/09/2016

How to cite this article: Zoubeidi H, Aydi Z, Daoud F, Rachdi I, Koubaa W, Jouini R et al. Kaposi sarcoma presenting as lymphadenopathy in an immunocompetent patient. EJCRIM 2016;3: doi:10.12890/2016_000493.

Conflicts of Interests: The Authors declare that there are no competing interests.

This article is licensed under a Commons Attribution Non-Commercial 4.0 License

\section{ABSTRACT}

Introduction: Kaposi's sarcoma (KS) is an angioproliferative disorder first described in 1872 by Moritz Kaposi. Four main clinical presentations of KS have been described: classic, endemic, iatrogenic and epidemic. KS involvement of the lymph nodes is extremely uncommon in the classical variant form, especially if it precedes the skin manifestations. We describe the case of an elderly HIV-negative patient presenting with lymphadenopathy who was found to have KS.

Case Report: A 67-year-old patient was admitted for exploration of polyadenopathies in the context of a general decline in health. Physical examination revealed an erythematosus left lower limb rash associated with angiomatous nodules and multiple lymphadenopathies. The diagnosis of erysipelas in the left leg was retained and the patient was treated with good evolution of the rash but persistence of the angiomatous nodules and the polyadenopathies. Skin and lymph node biopsies led to a diagnosis of KS. The patient is proposed for polychemotherapy.

Conclusion:KS must be suspected in lymphadenopathies despite the absence of typical cutaneous signs of the disease and in immunocompetent patients.

\section{LEARNING POINTS}

- Involvement of the lymph nodes is extremely uncommon in the classical variant form of Kaposi's sarcoma (KS).

- Human herpes virus-8 is an important cofactor in all forms of KS.

- Pathology and immunohistochemistry are key to diagnosing KS.

- KS must be suspected in lymphadenopathies without typical cutaneous signs of the disease and in immunocompetent patients.

\section{KEYWORDS}

Kaposi's sarcoma (KS), lymphadenopathy, human herpes virus-8 (HHV-8)

\section{INTRODUCTION}

Kaposi's sarcoma (KS) is an angioproliferative disorder characterized by proliferation of spindle-shaped cells, neoangiogenesis, inflammation and oedema, and categorized as an intermediate neoplasm due to the absence of conventional features of malignancy ${ }^{[1]}$. It was first described 
in 1872 by Moritz Kaposi ${ }^{[2]}$. Four distinct pathological forms of Kaposi's sarcoma (KS) are recognized: the classical variant, which occurs among elderly men of Jewish and East European origin; the endemic African form, which affects children, adolescents and adults and has a high frequency of extracutaneous manifestations; iatrogenic disease seen in organ transplant recipients undergoing immunosuppressive therapy; and epidemic disease, which is the most important opportunistic neoplasm occurring in human immunodeficiency virus type 1 (HIV-I)-infected individuals ${ }^{[3]}$. KS involvement of lymph nodes is frequently seen in the endemic African form and in the epidemic form in HIV-infected patients. In contrast, primary involvement of the lymph nodes in the classical variant form is extremely uncommon, especially if it precedes the skin manifestations, with only a few previously reported cases $^{[4-6]}$. We describe the case of an elderly HIV-negative patient presenting with lymphadenopathy who was found to have KS.

\section{CASE REPORT}

A 67-year-old man was admitted for exploration of shallow and deep polyadenopathies. His past medical history revealed hypertension and diabetes mellitus treated with metformin. The chief complaint was left inguinal lymphadenopathy without local inflammation where the lymph nodes had slowly enlarged over the previous 2 years in the context of gradually declining health. A previous lymph node biopsy had been inconclusive. Physical examination of the patient's lower left leg revealed a recent erythematosus, hot and painful rash associated with vesicles, blisters, purple papules and angiomatous nodules on the anteromedial surface of the left thigh and the lower two thirds of the left leg. There was associated lymphoedema of the left lower limb. Bilateral submandibular lymph nodes were nearly $2 \mathrm{~cm}$ in diameter, left supraclavicular lymph nodes nearly $1.5 \mathrm{~cm}$ in diameter, and bilateral axillary and bilateral inguinal lymph nodes 3-4 cm in diameter with a voluminous left popliteal lymphadenopathy. Investigations revealed an inflammatory syndrome with leucocytosis (WBC count 13,200 cells/ $\mathrm{mm} 3$, predominantly neutrophils), thrombocytosis (platelet count 560,000 cells/mm3), a C-reactive protein concentration of $108 \mathrm{mg} / \mathrm{l}$ and an erythrocyte sedimentation rate of $88 \mathrm{~mm}$. Renal and hepatic functions were normal. The blood smear was normal and tumour markers were negative. Chest x-ray did not show any particular abnormalities. The diagnosis of erysipelas of the left leg was retained and the patient was treated with penicillin $\mathrm{G}$ at a dose of 20 million IU/day with good evolution of the rash, but persistence of the angiomatous papulonodules and the polyadenopathies. Computed tomography showed hypervascularized cervical, thoracic and para-aortic polyadenopathies which were partly necrotic. Doppler ultrasound of the lower limbs showed voluminous, richly vascularized bilateral inguinal lymphadenopathy with mass effect on the common femoral artery and a voluminous left popliteal lymphadenopathy with a mass effect. Skin and lymph node biopsies were performed. The biopsy of the angiomatous nodules (Fig. 1) suggested KS. The lymph node biopsy (Fig. 2) indicated lymph node localization of KS. Immunohistochemistry using anti-human herpesvirus-8 (HHV-8) antibodies (Fig. 3) showed intense spotted nuclear labelling of tumour nuclei (Fig. 4). HIV, HBV and HCV serologies were negative. The diagnosis of KS was retained. The patient is proposed for polychemotherapy with adriamycin, vinblastine and bleomycin.

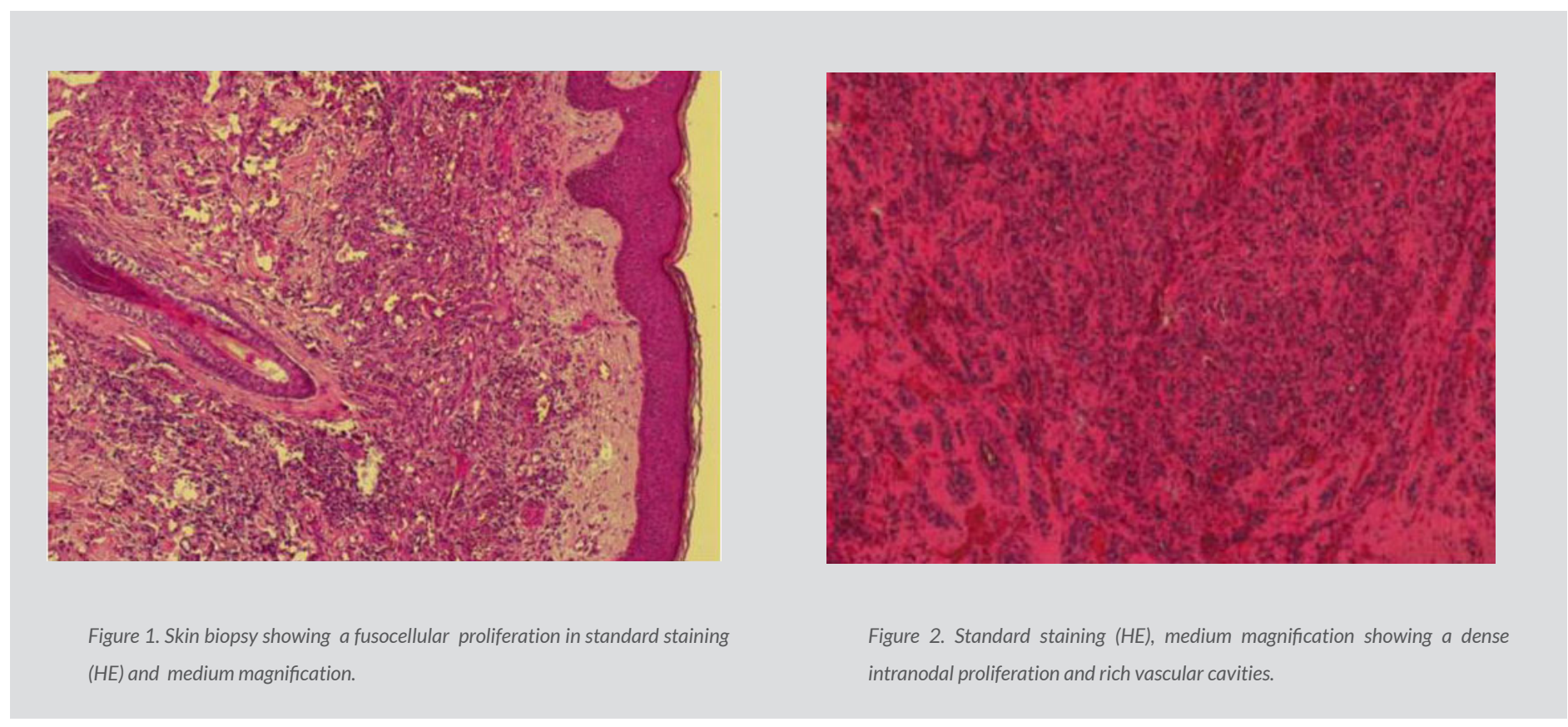




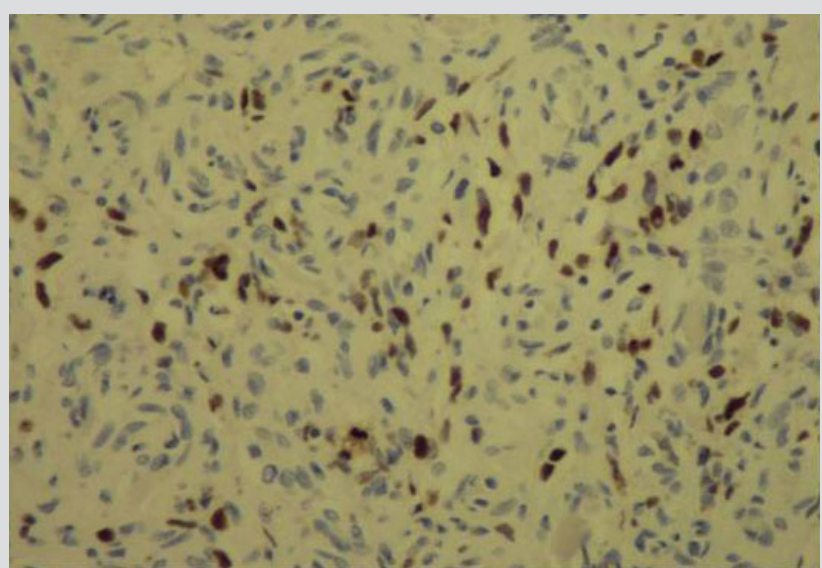

Figure 3. Positivity of Ac ant- HHV8 in Immunohistochemical study.

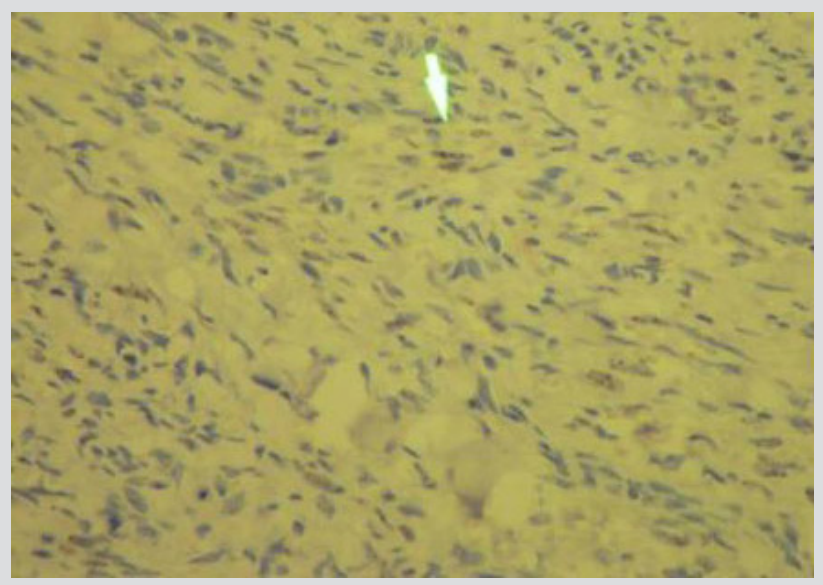

Figure 4. Nuclear Positivity of Ac anti-HHV8 in Immunohistochemical study in nuclei tumoral cells.

\section{DISCUSSION}

The clinical appearance of KS is classically described as pink, red, purple or violaceous macules, papules or raised plaques, although nodular and more distinctly neoplastic-looking forms have also been described ${ }^{[7]}$. While KS primarily affects mucocutaneous tissues, internal organs can also be involved. Our patient presented with the classical variant of KS which mostly affects the lower extremities. Primary involvement of the lymph nodes is uncommon and rarely reported, but preceding skin lesions can suggest the diagnosis. A few cases have been previously reported ${ }^{[4-6]}$. Lymphadenopathies are mostly seen in HIV-positive patients and are common in the epidemic form of KS.

The aetiology of KS is unknown. However, many epidemiological and environmental factors play a role in the development of the disease. In the last decade, evidence has suggested that infection with a recently discovered herpes virus, namely KS herpes virus or HHV-8, is responsible for all forms of $\mathrm{KS}^{[8]}$. While HHV-8 is asymptomatic in immunocompetent individuals, it could result in the development of neoplastic disorders, such as KS, in immunocompromised hosts. As a defect in the immune system is usually necessary, KS is typically associated with HIV infection. In our case, the patient is HIV negative and there is no evidence of immunodepression despite the HHV-8 positive tumour nuclei.

All clinical subtypes of KS have a similar histopathological appearance. Early lesions are characterised by a proliferation of small veins and capillaries around one or more dilated vessels. A pronounced mononuclear inflammatory cell infiltrate is often noted. There may also be perivascular proliferation of spindle cells, but cellular atypia is minima ${ }^{[9]}$. Treatment of KS can be systemic or local. The choice of therapy is determined by the extent of skin lesions, the systemic manifestations of the disease and the involvement of different organs. Therefore, staging of the tumour based on the clinical signs is recommended. Local treatments such as surgical excision or radiotherapy are mainly indicated for selected, small lesions, while systemic treatments include chemotherapy or biological therapy particularly with recombinant interferon- $\alpha$ due to its immunomodulating and anti-angiogenetic properties ${ }^{[10]}$. Systemic treatments are generally applied to disseminated and symptomatic forms. The optimal therapy for KS patients is still undecided, but new advances in understanding the pathogenesis of the disease may help to establish a standard protocol.

\section{CONCLUSION}

Our case report describes involvement of the lymph nodes in classical KS as the first manifestation of the disease before skin lesions. Consequently, the diagnosis of KS must be suspected when lymphadenopathies are present although typical cutaneous signs of the disease are absent, even in immunocompetent patients. Histology remains the key to diagnosis. 


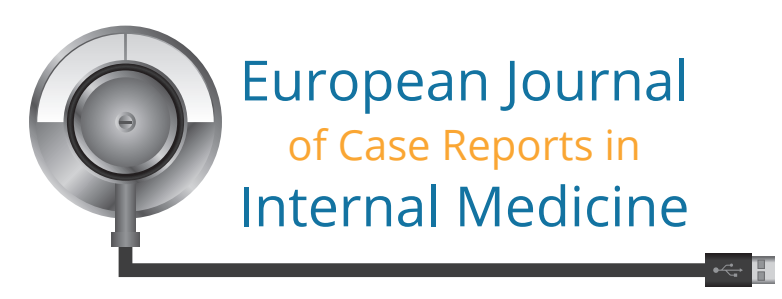

\section{REFERENCES}

1. Ramírez-Amador V, Martínez-Mata G, González-Ramírez I, et al. Clinical, histological and immunohistochemical findings in oral Kaposi's sarcoma in a series of Mexican AIDS patients. Comparative study. J Oral Pathol Med 2009;38:328-333.

2. Kaposi M. Idiopathisches multiples Pigmentsarkom der Haut. Arch Dermatol Syph 1872;4:265-273.

3. Armes J. A review of Kaposi's sarcoma. Adv Cancer Res 1989;53:73-86.

4. Ecklund RE, Valitis J. Kaposi sarcoma of lymph nodes: a case report. Arch Pathol 1962;74:224-229.

5. Ramos CV, Taylor HB, Hernandez BA, Tucker EF. Primary Kaposi's sarcoma of lymph nodes. Am J Clin Pathol 1976;66:998-1003.

6. Wang JC, Rosen Y, Goel PC, Teplitz H, Goldberg M, Friedman-Kien AE, et al. Kaposi's sarcoma presenting as lymphadenopathy in two HIV-negative elderly patients. Am J Med 1993;94:342-344.

7. Schwartz RA. Kaposi's sarcoma: an update. J Surg Oncol 2004;87:146-151.

8. Iscovich J, Boffetta P, Franceschi S, et al. Classic Kaposi sarcoma: epidemiology and risk factors. Cancer 2000;88:500-517.

9. Busi M, Altieri E, Ciorba A, Aimoni C. Auricular involvement of a multifocal non-AIDS Kaposi's sarcoma: a case report. Acta Otorhinolaryngol Ital 2014;34:146-149.

10. Sullivan RJ, Pantanowitz L, Dezube BJ. Targeted therapy for Kaposi sarcoma. Bio Drugs 2009;23:69-75. 